\title{
Adaptive Bayesian Contour Estimation: A Vector Space Representation Approach ${ }^{\star}$
}

\author{
José M. B. Dias \\ Instituto de Telecomunicações, \\ Instituto Superior Técnico, \\ 1049-001 Lisboa, PORTUGAL \\ bioucas@lx.it.pt
}

\begin{abstract}
We propose a vector representation approach to contour estimation from noisy data. Images are modeled as random fields composed of a set of homogeneous regions; contours (boundaries of homogeneous regions) are assumed to be vectors of a subspace of $L^{2}(T)$ generated by a given finite basis; B-splines, Sinc-type, and Fourier bases are considered. The main contribution of the paper is a smoothing criterion, interpretable as a priori contour probability, based on the Kullback distance between neighboring densities. The maximum a posteriori probability (MAP) estimation criterion is adopted. To solve the optimization problem one is led to (joint estimation of contours, subspace dimension, and model parameters), we propose a gradient projection type algorithm. A set of experiments performed on simulated an real images illustrates the potencial of the proposed methodology
\end{abstract}

\section{Introduction}

Boundary estimation/detection plays a key role in image analysis/understanding, pattern recognition, computer vision, computer graphics, and computer-aided animation. Although the approaches to contour estimation are numerous, most of them share the same spirit: contours are obtained through the maximization of objective functions composed of a prior term, that favors contours with some attributes (e.g., continuity, smoothness, elasticity, and rigidity), and a data term, that measures the adjustment to data.

As in many other fields, different aspects of contour estimation have been addressed either under the energy-minimization framework or under the Bayesian framework.

\subsection{Energy-Minimization Framework}

Under the energy viewpoint, data and prior terms are interpretable as external energy, which attracts the contour to the desired features, and internal energy (e.g., due to contour tension and rigidity), respectively. This perspective was introduced in the original work of Kass [16], where the concept of snake (or active

* This work was supported by the Portuguese PRAXIS XXI program, under project 2/2.1.TIT/1580/95. 
contour, or deformable model) was put out: "A snake is an energy-minimizing spline guided by internal constraint forces and influenced by image forces that pull it towards features such as lines and edges".

Since its introduction, the initial concept of active contour has been modified and improved in order to adapt it to different image classes and to overcome some of its drawbacks; namely, snake attraction by artifacts, snake degeneration, convergence and stability of the deformation process, myopia (i.e., use of image data only along the contour neighborhood), initialization, and model parameters estimation. References [3], [5], [11], [28] are illustrative examples of approaches to solve common problems with different snake techniques;

\subsection{Bayesian Framework}

Under the Bayesian viewpoint, the objective function referred above and its data and prior terms are interpretable as the posterior contour probability, the likelihood function associated to the observation mechanism, and the contour prior probability, respectively; since the sought contour maximizes the posterior probability, it is interpretable as the maximum a posteriori (MAP) estimate.

In many imaging problems (e.g., medical imaging, synthetic aperture radar, synthetic aperture sonar) the likelihood function can be derived from the knowledge of the generation mechanism [7], [10], rather than from other heuristic and common sense arguments. A statistical framework is therefore, in these cases, the correct choice.

Relevant advantages of the Bayesian approach are the following:

(a) it allows to include prior knowledge about the parameters to be estimated in a model-based fashion;

(b) it supplies an adequate framework for dealing with nuisance parameters (e.g., noise power, parameters distributions, blur coefficients).

\subsection{Prior and Contour Representation}

Contour representation and prior term, say $p_{c}$, are close issues that have received great attention, regardless of the viewpoint. In snake-type approaches the term $p_{c}$ is, typically, of the form

$$
p_{c}(\mathbf{c})=\int R(\mathbf{c}(t)) d t,
$$

where $R(\mathbf{c})$ measures the smoothness of the contour $\mathbf{c}$. Usually $R$ is the combination of norms of different derivatives [16]. In the Bayesian approach, Markov random fields have been used as a way of modelling contour smoothness [7], [10], [13].

The prior contour information can be imposed by appropriate selection of function $p_{c}$ and/or by introducing constraints on the set of admissible contours. For example, functional $R$ in (1) can be tailored in order to have continuous derivative contours. Another possibility is to find the solution in a constrained space; 
one can assume, for example, that contours belong to a parametrized family; i.e., $\mathbf{c}(t)=\mathbf{c}(t ; \boldsymbol{\alpha})$, where $\boldsymbol{\alpha}$ is defined in a given set $\Theta$. This is the case of the deformable parametrized models/templates (e.g., Fourier [11], spline [2], and wavelet descriptors [4]).

\subsection{Proposed Approach}

Herein we address contour estimation under the Bayesian setting. We assume that images are piece-wise homogeneous random fields, and that contours are the boundaries of open connected sets.

\section{Likelihood function}

The likelihood function is derived from the image generation mechanism. We assume that pixels within each homogeneous region are independent samples of a selected random variable. For example, coherent amplitude images (e.g., ultrasound and synthetic aperture radar and sonar images) are Rayleigh distributed [26], X-ray images are very well approximated with a Gaussian distribution [19], and nuclear and confocal microscopic images are Poisson distributed [22].

We take as hypothesis that the random variables associated with the image pixels are independent, i.e., we assume the so-called conditional independence property [14]. In an image system, this is a correct assumption if the resolution volumes contributing to different pixels are disjoint. This is approximately the case in most acquisition systems, since there is no information gain in acquiring extremely correlated neighboring data.

\section{Prior}

We assume that contours belong to a finite-dimensional subspace spanned by a given vector basis. Smoothness properties of contours are closely related to those of basis vectors and to the subspace dimension $K[6]$.

Roughly, the basis dimension determines the frequency content of contours. What should then be a suitable subspace dimention $K$ ? From the error projection point of view, $K$ should be as large as possible. However, as $K$ increases the subspace becomes less constrained and the estimated contours more noisy.

We tackle the estimation of the subspace dimension by assuming that contours $\mathbf{c}(t ; \boldsymbol{\alpha}, K)$ are random, with probability density function of the form

$$
p_{c}(\mathbf{c}(t ; \boldsymbol{\alpha}, k))=p_{K}(k) .
$$

The density $p_{K}$ is chosen to be a decreasing function of $K$, thus favoring smooth contours. The exact structure of $p_{K}$ is derived with basis on the estimate goodness.

The maximum a posteriori probability (MAP) estimation criterion is adopted. To solve the optimization problem one is led to, a gradient projection type algorhitm is used.

Dealing with contours as subspace elements is very appealing, namely due to the following: 
(a) it is a parametrized approach: given a subspace basis, a natural parametrization is the set of basis coefficients, which are normally much smaller than the basis dimension;

(b) given a generic contour $\mathbf{c} \in S$, the closest contour of $\mathbf{c}$ in a subspace of $S$ is given by the projection of $\mathbf{c}$ onto this subspace.

Fourier descriptors [11], B-splines [2], and wavelets [4] have already been proposed in the field of contour estimation. However, only work [11] explores the vector space perspective; namely, it introduces a minimum description length (MDL) [20] type principle for the determination the subspace dimension.

The MDL criterion, as applied in [11], is a smoothing criterion depending only on the subspace dimension $K$. The smoothing criterion herein proposed, besides depending on $K$, depends also on the Kullback distance between neighboring densities. This modification plays a key rule in assuring that estimated space dimension is, to a great extent, independent of the neighboring densities parameters.

The mais contribution of this work are the following:

(a) the study of the adequacy of subspaces generated by B-splines, Sinc-type, and Fourier bases to the smoothness contour modeling;

(b) the introduction of a criterion for the subspace dimension estimation based on the estimate robustness;

(c) the proposal of a complete adaptive scheme that iteratively estimates the contour, the distribution parameters, and the subspace dimension.

The paper is organized as follows. Section 2 addresses aspects of contour representation using B-splines, Sinc-type and Fourier, bases. Section 3 proposes two algorithms for contour estimation: the first assumes that the subspace dimension is know; the second estimates the subspace dimension jointly with the contours. Finally Section 4 presents results obtained with real data.

\section{Contour Representations and Subspaces}

Contours are closed periodic curves $c(t)=\{x(t), y(t)\}$, such that $c(t)=c(t+T)$. For notational convenience, assume that contours are defined in the complex plane $\mathcal{C}$, and, therefore, $x(t)$ and $y(t)$ are the real and imaginary parts of $c$, respectively.

We assume that $c \in L^{2}(T)$ (contour power over its period is finite). Since $L^{2}(T)$ is a separable Hilbert space [17], then there exist bases $\left\{\varphi_{n}(t)\right\}$, for $n=0,1, \ldots$, in $L^{2}(T)$, such that each vector $c \in L^{2}(T)$ is given by the linear combination

$$
c(t)=\sum_{n=0}^{\infty} \alpha_{n} \varphi_{n}(t) .
$$

For an orthoganal basis $\left\{\varphi_{n}(t)\right\}$, coefficients $\alpha_{n}$ are unique and given by

$$
\alpha_{n}=\left(c, \varphi_{n}\right) \equiv \frac{1}{T} \int_{0}^{T} c(t) \varphi_{n}^{*}(t) d t .
$$


Equality (3) is to be understood as a limit in norm.

In the proposed approach, contours are chosen to be elements of the subspace $S_{K} \equiv \operatorname{span}\left(\varphi_{0}, \ldots, \varphi_{K-1}\right)$ generated by the vector basis $\left\{\varphi_{n}(t)\right\}$, for $n=0,1, \ldots, K-1$. Each contour is then written as

$$
c(t ; \boldsymbol{\alpha})=\sum_{n=0}^{K-1} \alpha_{n} \varphi_{n}(t) .
$$

where $\boldsymbol{\alpha} \equiv\left\{\alpha_{0}, \ldots, \alpha_{K-1}\right\}$.

The contour smoothness constraint is enforced by adequate selection of the basis $\left\{\varphi_{n}\right\}$ and of the subspace dimension $K$. In this work we consider B-spline [6], Sinc-type, and Fourier bases.

\section{$2.1 \quad$ B-splines Basis}

Spline functions are piecewise polynomials [6], which have been widely used to represent contours and surfaces in computer graphics, computer vision, and signal and image processing [8], [9], [25], [24]. Given the set of so-called knots $\left\{t_{0}<t_{1}<\ldots<t_{k}\right\} \subset \Re$, a $m$-order spline is a piece-wise polynomial function defined on $\left[t_{0}, t_{k}\right]$, which are $C^{m-1}$ continuous on $\left[t_{m}, t_{k-m}\right]$. Given a knot sequence, the set of all splines which are $C^{m-1}$ continuous on $\left[t_{m}, t_{k-m}\right]$ is a linear space of dimension $(k-n)$. The family of so-called B-splines functions, generated by the Cox-deBoor recursion [6], is a basis for this linear space. For equispaced knots, the B-spline are named uniform, and given by

$$
\mathcal{B}_{i}^{m}(t)=\mathcal{B}_{0}^{m}\left(t-i T_{s}\right),
$$

where index $i$ denotes the $i$-th basis element, $T_{s}=t_{i+1}-t_{i}$, and

$$
\mathcal{B}_{0}^{m}(t)=\underbrace{\mathcal{B}_{0}^{0}(t) * \mathcal{B}_{0}^{0}(t) * \ldots \mathcal{B}_{0}^{0}}_{m \text { convolutions }},
$$

with

$$
\mathcal{B}_{0}^{0}= \begin{cases}1 & t_{i} \leq t<t_{i+1} \\ 0 & \text { otherwise. }\end{cases}
$$

Since we are interested in representing periodic curves, splines and their Bspline basis must be modified accordingly. For this purpose, define $\left\{\tilde{t}_{n}, n \in \mathcal{Z}\right\}$, with $\tilde{t}_{n}=t_{n} \bmod k$, as the periodic extension of the knot sequence $\left\{t_{0}<t_{1}<\right.$ $\left.\ldots<t_{k}\right\}$ [12]. The basis functions $\tilde{\mathcal{B}}_{i}^{m}(t)$ are now periodic extensions of $\mathcal{B}_{i}^{m}(t)$, with period $T=t_{k}-t_{0}$, given by

$$
\tilde{\mathcal{B}}_{i}^{m}(t)=\sum_{n=-\infty}^{\infty} \mathcal{B}_{i+n T}^{m}(t) .
$$

When using the spline representation, we assume that contours are elements of the subspace $S_{K} \equiv \operatorname{span}\left(\tilde{\mathcal{B}}_{0}^{m}, \tilde{\mathcal{B}}_{1}^{m}, \ldots, \tilde{\mathcal{B}}_{K-1}^{m}\right)$ and, therefore, $C^{m-1}$ continuous; the degree of smoothness is enforced by the subspace dimension $K$ : as the subspace dimension increases, contours becomes less constrained. 
B-splines exhibit local control: when representing curves as linear B-spline combinations, modifying a coefficient causes only a small part of the curve to change. This leads to simple an effective algorithms for computing displacements of the active contour under the influence of image forces.

In all examples herein presented we use $m=3$. The spline contours are therefore $C^{2}$ continuous. This a common choice in vision and computer graphics [9]. Nevertheless, the concepts to be presented apply to any $m$-order spline.

\subsection{Sinc-type Basis}

A natural way to impose smoothness is to constrain the curves to be $F$-bandlimited (i.e., having maximum frequency $F$ ). The set of $F$-bandlimited curves of finite energy is a linear space; the sequence $\left\{\mathcal{S}_{n}(t)\right\}=\left\{\mathcal{S}_{0}\left(t-n T_{s}\right)\right\}$, where $T_{s}=1 / 2 F$ and

$$
\mathcal{S}_{0}(t)=\sqrt{2 F} \frac{\sin 2 \pi F t}{2 \pi F t} \equiv \sqrt{2 F} \operatorname{sinc}(2 F t),
$$

is an orthonormal space basis. The projection of a curve $c(t)$ on $\mathcal{S}_{n}(t)$ is exactly $c\left(n T_{s}\right)$ (see, e.g., [27]).

In order to adapt basis elements $\mathcal{S}_{n}(t)$ to periodic curves, one should have $\left|\mathcal{S}_{0}(t)\right| \simeq 0$ for $|t| \geq T / 2$. Since $\mathcal{S}_{0}(t)$ goes to zero as $|t|^{-1}$, for $|t| \rightarrow \infty$, this might not be fulfilled, if $K=T / T_{s}$ is too small. To overcome this difficulty, we replace the basis function (9) with

$$
\mathcal{S}_{0}(t)=\sqrt{2 F} \frac{\sin 2 \pi F t}{2 \pi F t} \frac{\cos (2 \pi F t \rho)}{1-(4 \rho F t)^{2}} .
$$

Basis (10) is the impulse response of a raised cosine filter with a roll-off factor $\rho$ [15], which goes to zero as $|t|^{-3}$, for $|t| \rightarrow \infty$. Seting, for example, $\rho=0.4$, we can take, for most practical purposes, $\mathcal{S}_{0}(t) \simeq 0$ for $|t|>3 T_{s}$.

The basis $\left\{\mathcal{S}_{n}(t)\right\}=\left\{\mathcal{S}_{0}\left(t-n T_{s} /(1+\rho)\right)\right\}$, with $\mathcal{S}_{0}(t)$ given by (10) generates the space of $F(1+\rho)$-bandlimited functions. Therefore the smoothness of space elements is enforced by selecting $F$. Since the sampling interval $T_{s} /(1+\rho)$ must be equal to $T / K$ (i.e., an integer number of bases over $T$ ), the relation between $F$ and $K$ is

$$
F=\frac{1}{2(1+\rho)} \frac{K}{T}
$$

The periodic extension $\tilde{\mathcal{S}}_{0}^{m}(t)$ of $\mathcal{S}_{0}^{m}(t)$, with period $T$, is given by

$$
\tilde{\mathcal{S}}_{i}^{m}(t)=\sum_{n=-\infty}^{\infty} \mathcal{S}_{i+n T}^{m}(t)
$$

When using the bandlimited representation, we assume that contours are elements of the subspace $S_{K}=\operatorname{span}\left(\tilde{\mathcal{S}}_{0}, \tilde{\mathcal{S}}_{1}^{m}, \ldots, \tilde{\mathcal{S}}_{K-1}\right)$; the degree of smoothness is enforced by choosing $K$, which determines the maximum content frequency of contours according to (11).

As the B-spline basis, also the Sinc-type function (11), exhibits local control: the energy of $\tilde{\mathcal{S}}_{i}(t)$ is concentrated at $t=i T s$. 


\subsection{Fourier Basis}

The Fourier orthonornal basis is, probably, the representation most often used for periodic functions. In this representation the basis elements are given by

$$
\mathcal{F}_{n}(t)=e^{j \frac{2 \pi}{T} n t} \quad n \in \mathcal{Z} .
$$

With the Fourier representation, the most natural way of imposing smoothness is to restrict $L^{2}(T)$ to the finite subspace $S_{K}$ generated by $\left\{\mathcal{F}_{n}(t)\right\}$, for $n=-K+1, \ldots, 0, \ldots, K-1$. The generated contours are then given by

$$
c(t)=\sum_{n=-K+1}^{K-1} \alpha_{n} e^{j \frac{2 \pi}{T} n t} .
$$

Subspace $S_{K}$ is obtained by filtering $L^{2}(T)$ elements with an ideal low-pass filter of cut frequency $K / T$. As in the Sync-type basis, the smoothness degree is enforced by selecting the maximum contour content frequency. Contrarily to the B-spline and Sync-type representations, the Fourier basis does not exhibit local control.

\subsection{Contour Sampling and Fitting}

Due to the discrete nature of digital images, one frequently faces the problem of finding, in a given subspace $S_{K}$, the closest element of a set of discrete points. In other words, given $\mathbf{c} \equiv\left\{c\left(t_{0}\right), c\left(t_{1}\right), \ldots, c\left(t_{N-1}\right)\right\}$, find $\hat{\mathbf{c}} \in \mathcal{C}^{N}$, such that

$$
\hat{\mathbf{c}}=\arg \min _{\mathbf{w}(w), w \in S_{K}}\|\mathbf{c}-\mathbf{w}\|,
$$

where $\mathbf{w} \equiv\left\{w\left(t_{0}\right), w\left(t_{1}\right), \ldots, w\left(t_{N-1}\right)\right\}$.

In the fitting problem at hand, the set $\left\{t_{0}, t_{1}, \ldots, t_{N-1}\right\}$ and the period $T$ are not known. Herein we take $T=N$ and $t_{i}=i$, for $i=0,1 \ldots, N-1$, which is termed the uniform assignment strategy.

Define matrix B such that

$$
[\mathbf{B}]_{i j} \equiv \tilde{\varphi}_{j}\left(t_{i}\right), \quad i=0,1, \ldots, N-1, \quad j=0,1, \ldots, K-1,
$$

where $\tilde{\varphi}_{j}$ is one of the basis functions (8), (12), or (13).

In terms of matrix $\mathbf{B}$, minimization (15) is written as

$$
\hat{\mathbf{c}}=\arg \min _{\mathbf{w} \in \mathcal{R}(\mathbf{B})}\|\mathbf{c}-\mathbf{w}\|,
$$

where $\mathcal{R}(\mathbf{B})$ stands for the span generated by the columns of $\mathbf{B}$ (notation $\mathbf{B}_{k}$, when used, stresses that $k=\operatorname{dim}\left(\operatorname{span}\left(\mathbf{B}_{k}\right)\right)$. Using the Euclidian norm, and assuming that $K \leq N-1$, the projection (17) is given by

$$
\hat{\mathbf{c}}=\mathbf{B B}^{\#} \mathbf{c},
$$

with

$$
\mathbf{B}^{\#} \equiv\left(\mathbf{B}^{\mathbf{H}} \mathbf{B}\right)^{-1} \mathbf{B}^{H}
$$


being the pseudoinverse matrix of $\mathbf{B}$ [21]. Matrix $\mathbf{B}^{\#}$ also solves the following problem:

$$
\begin{aligned}
\boldsymbol{\theta} & =\arg \min _{\boldsymbol{\alpha} \in C^{K}}\|\mathbf{c}-\mathbf{B} \boldsymbol{\alpha}\| \\
& =\mathbf{B}^{\#} \mathbf{c},
\end{aligned}
$$

being, therefore, $\hat{\mathbf{c}}$ also given by $\mathbf{c}^{\#}=\mathbf{B} \boldsymbol{\theta}$.
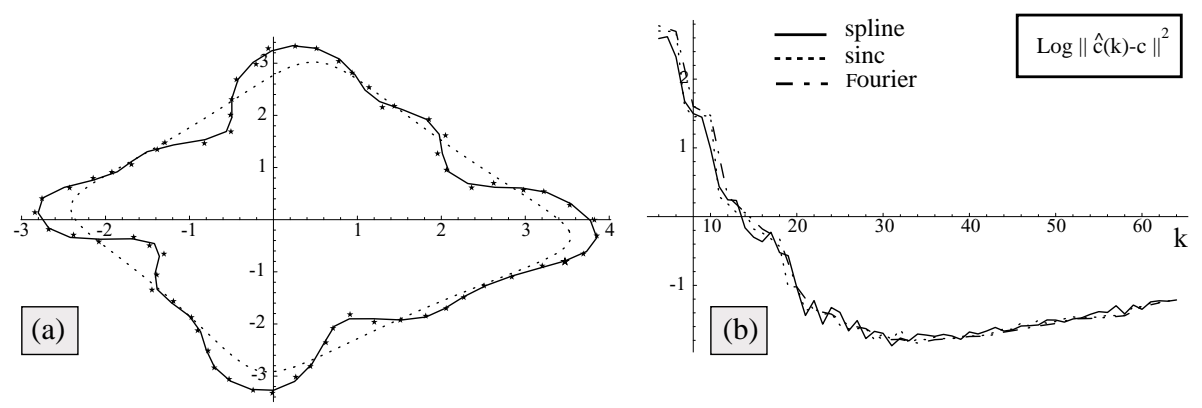

Fig. 1. (a) Projection of a noisy contour onto the subspace generated by Fourier basis. Stars represent the discrete contour to be projected, whereas doted and solid lines represent the projection onto $\mathcal{R}\left(\mathbf{B}_{10}\right)$ and $\mathcal{R}\left(\mathbf{B}_{30}\right)$, respectively. (b) Representation error for B-spline, Sinc-type, and Fourier bases.

Fig. 1(a) shows the projection of a hand traced contour contaminated with white noise, on the subspace generated by the Fourier basis. A complex zeromean Gaussian random variable with standard deviation of 0.1 was added to each coordinate; stars represent the discrete contour to be projected; doted and solid lines represent the projections onto $\mathcal{R}\left(\mathbf{B}_{10}\right)$ and $\mathcal{R}\left(\mathbf{B}_{30}\right)$, respectively. Projection onto $\mathcal{R}\left(\mathbf{B}_{10}\right)$ is clearly underfitted, while projection on $\mathcal{R}\left(\mathbf{B}_{30}\right)$ is nearly optimum. This can be perceived from the error projection plotted on Fig. 1(b). The minimum error occurs, for the three representations, roughly at $K=30$. For large values of $K$, the representation error increases, as the respective subspaces are now unable to smooth out the high frequency components of noise.

The similarity between the three representations, at least for the example presented, is evident. However, we would like to call attention to the following point: the representation error on the subspaces generated by Sinc-type and Fourier bases decreases until it reaches a minimum. This is not the case with the B-splines basis: the representation error, in this latter case, may increase, although slightly, with $K$. This behavior results from the non-nested structure of subspaces generated by the B-splines, whereas the subspaces generated by Sinctype and Fourier bases are nested: the linear space of $F$-banlimited functions contains all subspaces of $W$-bandlimited functions with $W \leq F$.

Subspaces having nested structure might be a desirable feature when the space dimension is unknown and it should be somehow estimated. 


\section{Image Generation Model}

Let $\mathbf{c}=\left\{c_{0}, \ldots, c_{N-1}\right\}$ be the boundary of a connected region $R_{1}$ of the plane and $R_{2}$ the set of points not in $R_{1}$. Denote $x_{i}$ as the image gray-level observed at $i$-th pixel, $\mathbf{x}=\left\{x_{i}\right\}$ as the set of image gray-levels, $p_{x}$ as the gray-level density, and $\boldsymbol{\psi}_{x}=\left\{\boldsymbol{\psi}_{1}, \boldsymbol{\psi}_{2}\right\}$ as the density parameters (i.e., $p_{x}\left(x_{i}\right)=p_{x}\left(x_{i} \mid \boldsymbol{\psi}_{1}\right)$ ) for $i \in R_{1}$ and $\left.p_{x}\left(x_{i}\right)=p_{x}\left(x_{i} \mid \boldsymbol{\psi}_{2}\right)\right)$ for $\left.i \in R_{2}\right)$. Since we take as hypothesis that the image random variables, conditioned to the contour, are independent, it follows that

$$
p_{\mathbf{x} \mid c}\left(\mathbf{x} \mid \mathbf{c}, \boldsymbol{\psi}_{x}\right)=\left(\prod_{i \in R_{1}} p_{x}\left(x_{i} \mid \boldsymbol{\psi}_{1}\right)\right)\left(\prod_{i \in R_{2}} p_{x}\left(x_{i} \mid \boldsymbol{\psi}_{2}\right)\right) .
$$

According to the proposed approach, contour $\mathbf{c}$ belongs to the subspace $\mathcal{R}\left(\mathbf{B}_{K}\right)$, being therefore given by $\mathbf{c}=\mathbf{B}_{K} \boldsymbol{\alpha}$, for $\boldsymbol{\alpha} \in \mathcal{C}^{K}$. Subscript $K$ will occasionally be omitted.

\subsection{Bayesian Approach to Contour Estimation}

In accordance with the rationale already exposed, we assume that contours $\mathbf{c}(K)=\mathbf{c}(K, \boldsymbol{\alpha})$ are random vectors with probability density function given by

$$
p_{c}(\mathbf{c}(k))=p_{K}\left(k \mid \psi_{c}\right),
$$

where $\boldsymbol{\psi}_{c}$ denotes a parameter vector of $p_{K}$. Hence, the MAP estimate of the pair $(\mathbf{c}, K)$ is

$$
(\hat{\mathbf{c}}, \hat{K})=\arg \max _{k, \mathbf{c} \in \mathcal{R}\left(\mathbf{B}_{k}\right)} p_{\mathbf{x} \mid c}\left(\mathbf{x} \mid \mathbf{c}, \boldsymbol{\psi}_{x}\right) p_{K}\left(k \mid \boldsymbol{\psi}_{c}\right)
$$

\subsection{Known Space Dimension}

Consider now that $K$ is know. The MAP estimate (24) is, under this condition, simply the maximum likelihood (ML) contour estimate given by

$$
\hat{\mathbf{c}}=\arg \max _{\mathbf{c} \in \mathcal{R}\left(\mathbf{B}_{k}\right)} L_{\mathbf{x} \mid c}\left(\mathbf{x} \mid \mathbf{c}, \boldsymbol{\psi}_{x}\right)
$$

where $L_{\mathbf{x} \mid c}\left(\mathbf{x} \mid \mathbf{c}, \boldsymbol{\psi}_{x}\right) \equiv \log p_{\mathbf{x} \mid c}\left(\mathbf{x} \mid \mathbf{c}, \boldsymbol{\psi}_{x}\right)$ is the loglikelihood function.

To compute $\hat{\mathbf{c}}$, we implement an ascent type iterative algorithm that, in the $t$-th iteration, implements the following steps:

1. determine, in the unconstraint space $\mathcal{C}^{N}$, a contour displacement $\Delta \mathbf{c}^{(t)}$ that increases $L_{\mathbf{x} \mid c}\left(\mathbf{x} \mid \mathbf{c}, \boldsymbol{\psi}_{x}\right)$

2. project $\mathbf{c}^{(t)}+\Delta \mathbf{c}^{(t)}$ onto the constrained subspace $\mathcal{R}(\mathbf{B})$, thus obtaining $\mathbf{c}^{(t+1)}$.

The displacement $\Delta \mathbf{c}^{(t)}$ is computed along orthogonal lines, as schematized in Fig. 2. Underlying this choice is the fact that the gradient of $L_{\mathbf{c} \mid c}(\mathbf{x} \mid \mathbf{c})$, computed with respect to $\mathbf{c}$, is orthogonal to the tangent vector $d \mathbf{c} / d t$ [23]. 

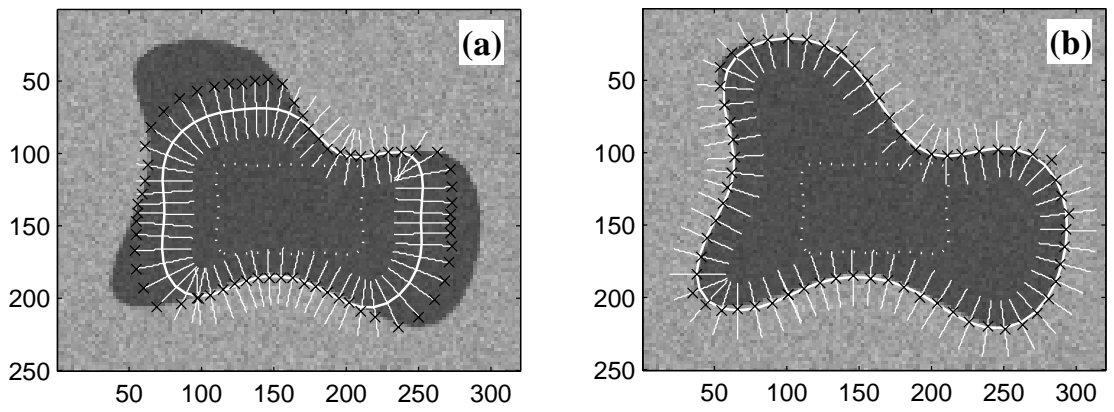

Fig. 2. Contour displacements computed along orthogonal lines. Crosses show the maximum of the loglikelihood function, along each orthogonal line. The doted line denotes the initial contour.

To prevent contours to be self intercepting, the orthogonal lines should be not too large. Work [23] proposes a technique for selecting long range orthogonal curves that do not intercept each other. Herein, however, we do not follow the mentioned technique, since it is not suited to our setting.

When the vector $\psi_{x}$ is not known, we determine ML estimate of vector $\left(\mathbf{c}, \psi_{x}\right)$ according to

$$
\left(\hat{\mathbf{c}}, \hat{\boldsymbol{\psi}}_{x}\right)=\arg \max _{\mathbf{c} \in \mathcal{R}(\mathbf{B}), \boldsymbol{\psi}_{x}} L_{\mathbf{x} \mid c}\left(\mathbf{x} \mid \mathbf{c}, \boldsymbol{\psi}_{x}\right) .
$$

To compute $\left(\hat{\mathbf{c}}, \hat{\boldsymbol{\psi}_{x}}\right)$, given by (26), the following iterative scheme is implemented:

Initialization: set $\mathbf{c}^{(0)}, \boldsymbol{\psi}_{x}^{(0)}$, and $\delta$

DO

step 1: $\Delta \mathbf{c}^{(t)}=\arg \max _{\mathbf{u} \in \mathcal{O}\left(\mathbf{c}^{(t)}\right)} L_{\mathbf{x} \mid c}\left(\mathbf{x} \mid \mathbf{c}^{(t)}+\mathbf{u}, \boldsymbol{\psi}_{x}^{(t)}\right)$

where $\mathcal{O}(\mathbf{c}) \subset \mathcal{C}^{N}$ is the set of points defining

orthogonal displacements to the contour $\mathbf{c}$

step 2: $\mathbf{c}^{(t+1)}=\mathbf{c}^{(t)}+\mathbf{B} \Delta \mathbf{c}^{(t)}$

step 3: $\boldsymbol{\psi}_{x}^{(t+1)}=\arg \max _{\boldsymbol{\psi}} L_{\mathbf{x} \mid c}\left(\mathbf{x} \mid \mathbf{c}^{(t+1)}, \boldsymbol{\psi}_{x}\right)$

step 4: $\Delta L=L_{\mathbf{x} \mid c}^{(t+1)}-L_{\mathbf{x} \mid c}^{(t)}$

While $|\Delta L| \geq \delta$.

Vector $\boldsymbol{\psi}_{x}^{(t+1)}$ can be written in terms of regions $R_{1}$ and $R_{2}$ as

$$
\psi_{1}^{(t)}=\arg \max _{\psi_{1}} \sum_{i \in R_{1}^{(t)}} L_{x \mid c}\left(x_{i} \mid \psi_{1}\right)
$$




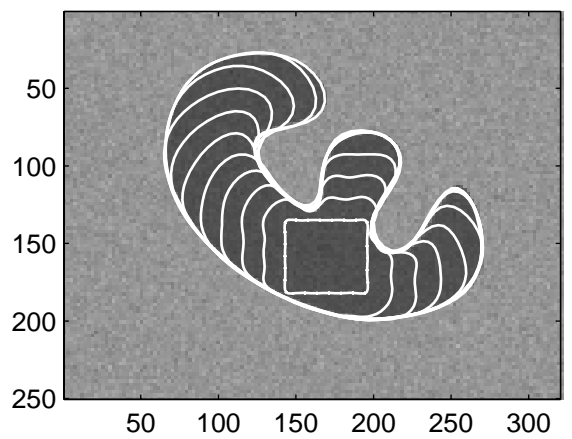

Fig. 3. Sequence of contour estimates produced by the proposed technique. The inner square represents the initial contour.

$$
\psi_{2}^{(t)}=\arg \max _{\psi_{1}} \sum_{i \in R_{2}^{(t)}} L_{x \mid c}\left(x_{i} \mid \psi_{2}\right)
$$

Expressions (27) and (28) depend on the particular structure of $p_{x}$. For example, for Gaussian densities with mean $\mu$ and variance $\sigma^{2}$, estimates of $\psi_{i} \equiv\left\{\mu_{i}, \sigma_{i}^{2}\right\}$, for $i=1,2$, are given by the sample mean and sample variance within the respective region.

Fig. 2, parts (a) and (b), displays estimates $\widehat{\mathbf{c}}^{(2)}$ and $\widehat{\mathbf{c}}^{(5)}$, respectively, of a Gaussian image with parameters $\left\{\mu_{1}=60, \sigma_{1}=15\right\}$ and $\left\{\mu_{2}=160, \sigma_{2}=30\right\}$. The boundary is obtained from a hand traced contour followed by projection onto $\mathcal{R}\left(\mathbf{B}_{7}\right)$.

Fig. 3 shows a sequence of contours estimates produced by the proposed algorithm. The long range nature of the external forces pulls the contour outwards as it was under an expansion force.

Fig. 4, part (a) and (b), displays two final estimates of Gaussian images with parameters $\boldsymbol{\psi}_{(a)}=\left\{\left(\mu_{1}=80, \sigma_{1}=15\right),\left(\mu_{2}=160, \sigma_{1}=30\right)\right\}$ and $\boldsymbol{\psi}_{(b)}=\left\{\left(\mu_{1}=\right.\right.$ $\left.\left.100, \sigma_{1}=15\right),\left(\mu_{2}=100, \sigma_{1}=30\right)\right\}$. The estimated contours are nearly the true ones, even for image (b), which exhibits no contrast at all (i.e., $\mu_{1}=\mu 2$ ).

\subsection{Unknown Space Dimension}

Consider now that the space dimension is unknown and, consequently, it is also to be estimated jointly with the contour. Noting that $\mathbf{c}=\mathbf{c}(K)$, and according to expression (24), the MAP estimate of the space dimension is given by

$$
\hat{K}=\arg \max _{k}\left\{L_{K}\left(k \mid \boldsymbol{\psi}_{c}\right)+\arg \max _{\mathbf{c} \in \mathcal{R}\left(\mathbf{B}_{K}\right)} L_{\mathbf{x} \mid c}\left(\mathbf{x} \mid \mathbf{c}, \boldsymbol{\psi}_{x}\right)\right\},
$$

where $L_{K}\left(k \mid \psi_{c}\right) \equiv \log p_{K}\left(k \mid \psi_{c}\right)$. The estimated contour is the ML solution studied in the previous section, with the space dimension set to $\hat{K}$. 

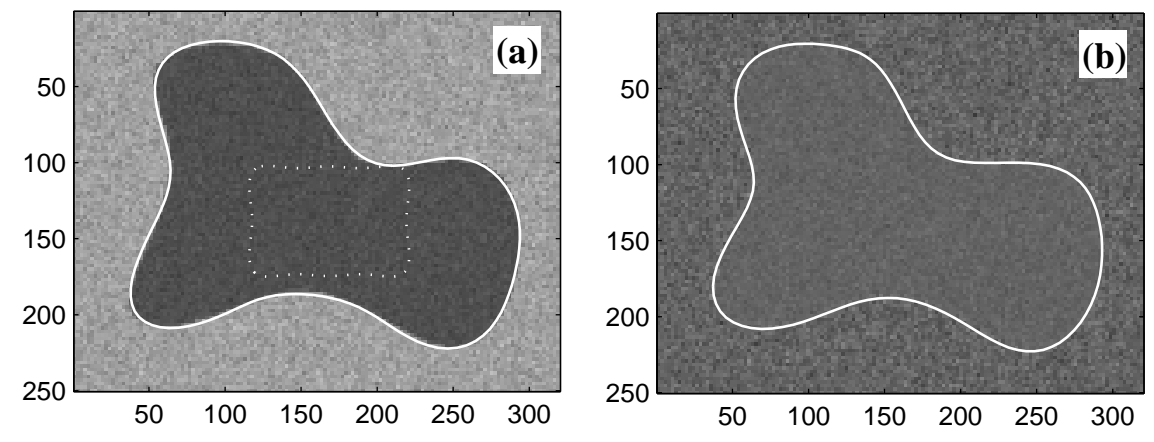

Fig. 4. Illustration of performance at very low contract: gray-levels in image (b) have the same mean value in both regions; in spite of this, the estimated contour is identical to the one estimated from image (a), which has high contrast.

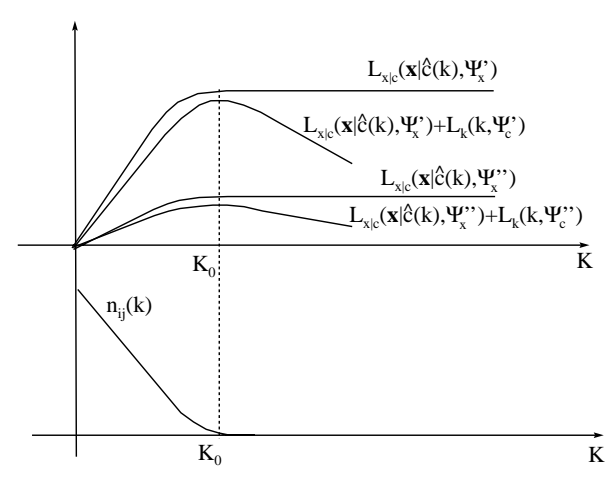

Fig. 5. Behavior of the loglikelihood function for two different vector parameters.

As in any Bayesian approach, the prior term must be specified. The first thought that could come to mind is to assume that $p_{K}\left(k \mid \boldsymbol{\psi}_{c}\right)$ is uniformly distributed for $K_{\min } \leq K \leq K_{\max }$; the estimate of $(\mathbf{c}, K)$ would therefore be interpretable as a ML estimate. Unfortunately, this attempt would fail. The reason is the following: due the nested nature of parameter spaces $\left(\mathcal{R}\left(\mathbf{B}_{K}\right) \subset \mathcal{R}\left(\mathbf{B}_{K+1}\right)\right)$, the loglikelihood function $L_{\mathbf{x} \mid c}\left(\mathbf{x} \mid \hat{\mathbf{c}}(\hat{K}), \boldsymbol{\psi}_{x}\right)$ will be a monotonically (or at least nondecreasing) function of $K$, so it will reach its maximum at $K_{\max }$.

The problem of choosing the order of competing models of different dimensions is termed a model order selection problem. Among the approaches that have been suggested to this problem, the Akaike information criterion (AIC) [1], and the minimum description length (MDL) [20] have gained popularity. Work [11], also on contour estimation, applies the MDL principle to derive the term $L_{K}\left(k \mid \psi_{c}\right)$ of $(29)$. 

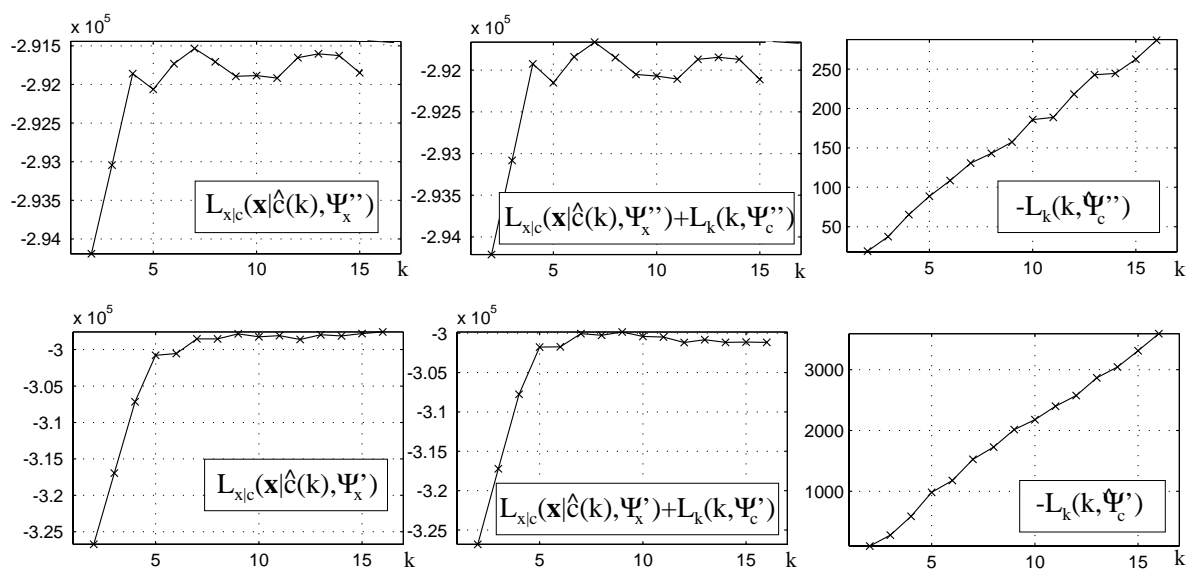

Fig. 6. Loglikelihood and prior behavior for the images shown in Fig. 7.

In this work we propose the prior

$$
p_{K}\left(k \mid \boldsymbol{\psi}_{c}\right)=\frac{1}{Z} e^{-\alpha k}, \quad \alpha>0,
$$

where $Z$ is a normalizing constant, and $\alpha$ is given by

$$
\alpha=\frac{n_{c}}{4} \mu D\left(\boldsymbol{\psi}_{\mathbf{1}}, \boldsymbol{\psi}_{\mathbf{2}}\right),
$$

where $n_{c}$ is the number of contour pixels, $\mu \simeq 0.1$, and

$$
D\left(\boldsymbol{\psi}_{\mathbf{1}}, \boldsymbol{\psi}_{\mathbf{2}}\right) \equiv D\left(\boldsymbol{\psi}_{\mathbf{1}} \| \boldsymbol{\psi}_{\mathbf{2}}\right)+D\left(\boldsymbol{\psi}_{\mathbf{2}} \| \boldsymbol{\psi}_{\mathbf{1}}\right),
$$

is the symmetric Kullback distance [18] and $D\left(\boldsymbol{\psi}_{\mathbf{1}} \| \boldsymbol{\psi}_{\mathbf{2}}\right)$ the Kullback distance [18] between densities $p_{x}\left(x_{i} \mid \psi_{2}\right)$ and $p_{x}\left(x_{i} \mid \psi_{2}\right)$ given by

$$
D\left(\boldsymbol{\psi}_{\mathbf{1}} \| \boldsymbol{\psi}_{\mathbf{2}}\right)=E_{\boldsymbol{\psi}_{\mathbf{1}}} \log \frac{p_{x}\left(x_{i} \mid \boldsymbol{\psi}_{\mathbf{1}}\right)}{p_{x}\left(x_{i} \mid \boldsymbol{\psi}_{\mathbf{2}}\right)}
$$

The derivation of prior (30) is out of the scope of this paper. We present, however, an informal justification. Aiming at this purpose, define

$$
L_{c \mid \mathbf{x}}(k \mid \mathbf{x}, \boldsymbol{\psi})=L_{K}\left(k \mid \boldsymbol{\psi}_{c}\right)+\arg \max _{\mathbf{c} \in \mathcal{R}\left(\mathbf{B}_{K}\right)} L_{\mathbf{x} \mid c}\left(\mathbf{x} \mid \mathbf{c}, \boldsymbol{\psi}_{x}\right) .
$$

Define also the sets $A_{12}$ and $A_{21}$ containing pixel indexes wrongly classified: in the first case region 1 has been detected, whereas in the second case region 2 has been detected. Assume that the true dimension space is $k_{0}$ and introduce

$$
\Delta L_{c \mid \mathbf{x}}(k) \equiv L_{c \mid \mathbf{x}}(k \mid \mathbf{x}, \boldsymbol{\psi})-L_{c \mid \mathbf{x}}\left(k_{0} \mid \mathbf{x}, \boldsymbol{\psi}\right)
$$


The difference $\Delta L_{c \mid \mathbf{x}}(k)$ can be written in terms of $A_{12}(k)$ and $A_{21}(k)$ as

$$
\begin{aligned}
\Delta L_{c \mid \mathbf{x}}(k)= & \sum_{i \in A_{12}(k)} \log \frac{p_{x}\left(x_{i} \mid \boldsymbol{\psi}_{\mathbf{1}}\right)}{p_{x}\left(x_{i} \mid \boldsymbol{\psi}_{\mathbf{2}}\right)}+ \\
& \sum_{i \in A_{21}(k)} \log \frac{p_{x}\left(x_{i} \mid \boldsymbol{\psi}_{\mathbf{2}}\right)}{p_{x}\left(x_{i} \mid \boldsymbol{\psi}_{\mathbf{1}}\right)}+\Delta L_{K}(k),
\end{aligned}
$$

where

$$
\Delta L_{K}(k) \equiv L_{K}\left(k \mid \boldsymbol{\psi}_{c}\right)-L_{K}\left(k_{0} \mid \boldsymbol{\psi}_{c}\right) .
$$

A lengthy manipulation of (36), and a few weak assumptions, lead to

$$
E\left\{\Delta L_{c \mid \mathbf{x}}(k) \| k_{0}\right\}=-n_{12}(k) D\left(\boldsymbol{\psi}_{\mathbf{1}}, \boldsymbol{\psi}_{\mathbf{2}}\right)+\Delta L_{K}(k),
$$

where $n_{i j}(k) \equiv \# A_{i j}(k)$ is the number of elements of $A_{i j}$.

The interpretation of $(38)$ is clear: term $n_{12}(k) D\left(\boldsymbol{\psi}_{\mathbf{1}}, \boldsymbol{\psi}_{\mathbf{2}}\right)$ tends to zero as the the number of missclassified pixels tends to zero. The vanishing rate is proportional to the symmetric Kullback distance $D\left(\boldsymbol{\psi}_{\mathbf{1}}, \boldsymbol{\psi}_{\mathbf{2}}\right)$.

Fig. 5 schematizes the behavior of the loglikelihood function $L_{\mathbf{x} \mid c}\left(\mathbf{x} \mid \mathbf{c}, \boldsymbol{\psi}_{x}\right)$ and of the prior term $L_{K}\left(k \mid \boldsymbol{\psi}_{c}\right)$, for two vector parameters $\boldsymbol{\psi}_{x}^{\prime}$ and $\boldsymbol{\psi}_{x}^{\prime \prime}$. When $n_{i j}(k)$ approaches zero, $L_{\mathbf{x} \mid c}\left(\mathbf{x} \mid \mathbf{c}, \boldsymbol{\psi}_{x}\right)$ approaches a constant. By adding an appropriate prior term $L_{K}\left(k, \psi_{c}^{\prime}\right)$ to the loglikelihood function, a maximum is obtained at $k=k_{0}$. For the second vector parameter $\psi_{x}^{\prime \prime}$, the increasing rate of $L_{\mathbf{x} \mid c}\left(\mathbf{x} \mid \mathbf{c}, \psi_{x}^{\prime \prime}\right)$ is slower than $L_{\mathbf{x} \mid c}\left(\mathbf{x} \mid \mathbf{c}, \boldsymbol{\psi}_{x}^{\prime}\right)$. If the prior term $L_{K}\left(k, \psi_{c}^{\prime}\right)$ was used, a maximum would be obtained for $k<K_{0}$.
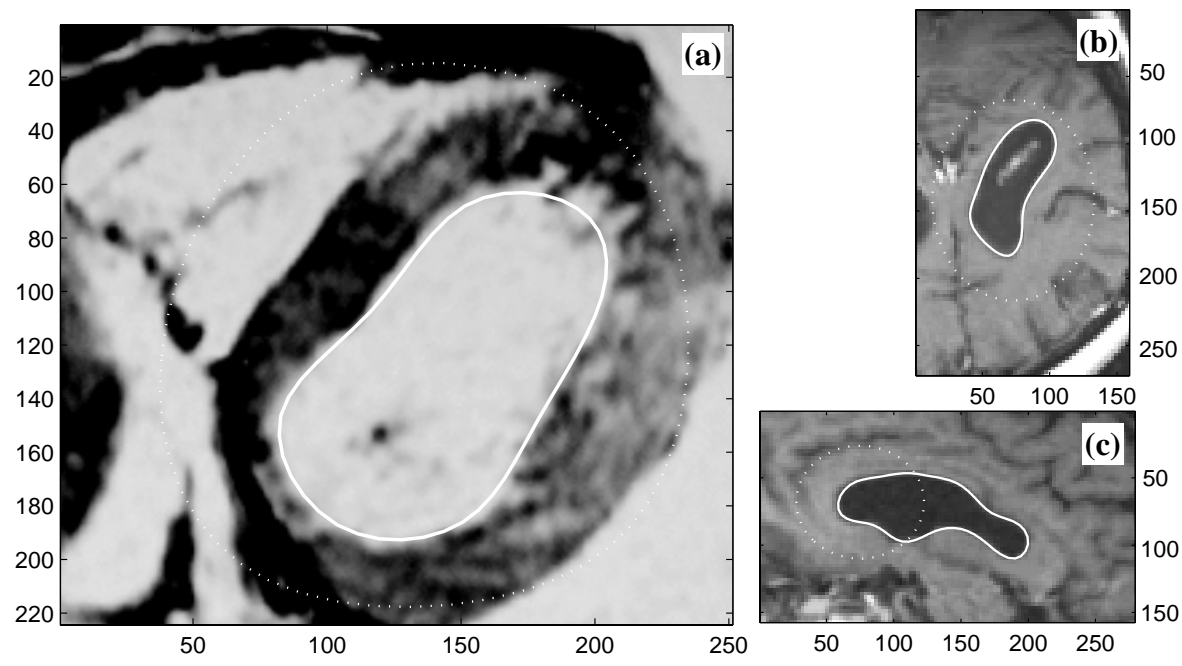

Fig. 7. Magnetic resonance images: (a) heart and (b,c) brain. 
Fig. 6 displays the loglikelihood and prior behavior for images shown in Fig. 4: the left column plots data from part (b), while the right column plots data from part (a). For both cases the maximum of $L_{\mathbf{x} \mid c}+L_{k}$ is obtained for $k=7$. However, the loglikelihood function $L_{\mathbf{x} \mid c}\left(\mathbf{x} \mid \mathbf{c}, \boldsymbol{\psi}_{x}^{\prime \prime}\right)$ grows much slower than $L_{\mathbf{x} \mid c}\left(\mathbf{x} \mid \mathbf{c}, \boldsymbol{\psi}_{x}^{\prime}\right)$, approximately by a factor of 12 , determined in the interval $k \in\{2,3,4,5\}$. For the maximizer $k$ to be the same, the prior term $L_{K}\left(k, \psi_{c}^{\prime \prime}\right)$ must grow slower than $L_{K}\left(k, \psi_{c}^{\prime}\right)$ by the same factor. This is, with great approximation, what happens.

The symmetric Kullback distance, for Gaussian distributions, is given by

$$
D\left(\boldsymbol{\psi}_{\mathbf{1}}, \boldsymbol{\psi}_{\mathbf{2}}\right)=\frac{\left(\sigma_{1}^{2}-\sigma_{1}^{2}\right)^{2}+\left(\sigma_{1}^{2}+\sigma_{1}^{2}\right)\left(\mu_{1}-\mu_{2}\right)^{2}}{2 \sigma_{1}^{2} \sigma_{2}^{2}} .
$$

Noting that the parameters associated with images displayed in Fig. 6 are $\boldsymbol{\psi}_{x}^{\prime \prime}=$ $\boldsymbol{\psi}_{x}(a)=\left\{\left(\mu_{1}=80, \sigma_{1}=15\right),\left(\mu_{2}=160, \sigma_{2}=30\right)\right\}$ and $\boldsymbol{\psi}_{x}^{\prime}=\boldsymbol{\psi}_{x}(b)=\left\{\left(\mu_{1}=\right.\right.$ $\left.\left.100, \sigma_{1}=15\right),\left(\mu_{2}=100, \sigma_{2}=30\right)\right\}$, it follows that $D\left(\boldsymbol{\psi}_{1}^{\prime \prime}, \boldsymbol{\psi}_{2}^{\prime \prime}\right) / D\left(\boldsymbol{\psi}_{1}^{\prime}, \boldsymbol{\psi}_{2}^{\prime}\right) \simeq 10$, in accordance with the experimental results.

Fig. 7 shows estimated contours over real magnetic resonance images: (a) heart and $(b, c)$ brain. The Gaussian model and the Fourier basis was used. The estimated space dimensions are 4,6 , and 7 , which are in agreement with the contours frequency content.

We stress the methodology robustness with respect to image nonhomogeneities and poor contour initializations.

\section{Concluding Remarks}

This paper introduced a novel adaptive methodology to contour estimation from noisy images. The approach was Bayesian: images were modeled as as a set of homogeneous regions, in a statistical sense. Contours were assumed to be vectors of a subspace generated by a finite basis: B-splines, Fourier, and Sinc-type bases were studied. It was concluded that Fourier, and Sinc-type bases were better suited to the proposed technique due to its nesting property.

A relevant contribution of the paper was on the contour prior design. By parametrizing the a priori probability density function with the symmetric Kullback distance between densities of each homogeneous region, the proposed algorithm produces meaningful estimates.

The proposed scheme is completely adaptive; all model parameters are estimated jointly with the contour. Results obtained with simulated and real data show the adequacy of the proposed approach.

\section{References}

1. H. Akaike. A new look at statistical model identification. IEEE Transactions on Automatic Control, AC-19:716-723, 1974.

2. A. Amini, R. Curwen, and J. Gore. Snakes and splines for tracking non-rigid heart motion. In Proc. European Conf. on Com. Vision - ECCV'96, pages 251-261, Cambrige, 1996. Springer Verlag. 
3. A. Amini, T. Weymouth, and R. Jain. Using dynamic programming for solving variational problems in vision. IEEE Trans. on Pattern Analysis and Machine Intelligence, PAMI-12(9):855-867, Sep. 1990.

4. G. Chuang and C. Kuo. Wavelet description of planar curves: theory and applications. IEEE Trans. on Image Proc., 5:56-70, 1996.

5. L. Cohen and I. Cohen. Finite-element methods for active contour models and baloons for 2D and 3D images. IEEE Trans. Pattern Anal. Machine Intell., 15:1131-1147, 1993.

6. C. de Boor. A Practical Guide to Splines. Springer Verlag, New York, 1978.

7. J. Dias and J. Leitão. Wall position and thickness estimation from sequences of echocardiograms images. IEEE Transactions on Medical Imaging, 15:25-38, Feb. 1996.

8. P. Dierckx. Curve and Surface Fitting with Splines. Oxford University Press, Oxford, 1993.

9. G. Farin. Curves and Surface Fitting for Computer Aided Geometrical Design. Oxford University Press, Oxford, 1993.

10. M. Figueiredo and J. Leitão. Bayesian estimation of ventricular contours in angiographic images. IEEE Trans. Med. Imag., MI-11(3):416-429, September 1992.

11. M. Figueiredo, J. Leitão, and A. K. Jain. Adaptive b-splines and boundary estimation. In Proc. of the IEEE Comp. Soc. Conf. on Com. Vision and Patt. Rec. CVPR'97, pages 724-730, San Juan (PR), 1997.

12. M. Flickner, J. Hafner, E. Rodriguez, and J. Sanz. Periodic quasi-orthogonal spline basis and applications to least square fitting of digital images. IEEE Trans. on Image Proc., 5:71-88, 1996.

13. N. Friedland and D. Adam. Automatic ventricular cavity boundary detection from sequential ultrasound images using simulated annealing. IEEE Transactions on Medical Imaging, MI-8(4):344-353, December 1989.

14. S. Geman and D. Geman. Stochastic relaxation, Gibbs distribution and the Bayesian restoration of images. IEEE Trans. Pattern Analysis and Machine Intelligence, PAMI-6(6):721-741, Nov. 1984.

15. S. Haykin. Communication Systems. John Wiley \& Sons, New York, 1983.

16. M. Kass, A. Witkin, and D. Terzopoulos. Snakes: Active countour models. Intern. Journal of Comp. Vision, 1:259-268, 1987.

17. E. Kreyszig. Introduction to Functional Analysis with Applications. John Wiley \& Soons, New York, 1978.

18. S. Kullback. Information Theory and Statistics. Peter Smith, 1978.

19. A. Macovski. Medical Imaging Systems. Prentice-Hall, Englewood Cliffs, NJ, 1983.

20. J. Rissanen. Modeling by shortest data description. Automatica, 14:465-471, 1978.

21. L. Scharf. Statistical Signal Processing. Detection, Estimation and Time Series Analysis. Addison-Wesley, New York, 1991.

22. D. L. Snyder. Random Point Process. John Wiley \& Sons, New York, 1975.

23. H. Tagare. Deformable 2-D template maching using orthogonal curves. IEEE Trans. Medical Imaging, 1:108-117, 1997.

24. M. Unser, A. Aldroubi, and M. Eden. B-spline signal processing: part i-efficient design and applications. IEEE Trans. Signal Process., 41(2):834-847, 1993.

25. M. Unser, A. Aldroubi, and M. Eden. B-spline signal processing: part i-theory. IEEE Trans. Signal Process., 41(2):821-833, 1993.

26. R.F. Wagner, M.F. Insana, and D.G.Brown. Statistical properties of radiofrequency and envelope detected signals with applications to medical ultrasound. J. Opt. Soc. Am., 4:910-922, May 1987.

27. A. Zayed. Advances in Shannon's Sampling Theory. CRC Press, 1993.

28. S. Zhu and A. Yuille. Region competion: Unifying snakes, region growing, energy/Bayes/MDL for multi-band image segmentation. IEEE Trans. Pattern Anal. Machine Intell., 18:884-900, 1996. 